\title{
The role of cervical length in predicting the success of induction of labour
}

\author{
A Amupala, ${ }^{1} \mathrm{MB} \mathrm{ChB}, \mathrm{Dip}$ Obst (SA); T Cronje, ${ }^{2} \mathrm{MSc}$; \\ S Adam, ${ }^{1}$ MB ChB, FCOG (SA), MMed (O\&G), Cert Mat Fetal Med (SA), PGCHE, PhD \\ ${ }^{1}$ Department of Obstetrics and Gynaecology, Faculty of Health Sciences, University of Pretoria, South Africa \\ ${ }^{2}$ Department of Statistics, Faculty of Natural and Agricultural Sciences, University of Pretoria, South Africa
}

Corresponding author: S Adams (sumaiya.adam@up.ac.za)

Background. Induction of labour (IOL) is one of the most common procedures conducted in obstetric practice. Several scoring models are used to predict the probability of successful IOL, most notably the modified Bishop score. Cervical length measured by transvaginal ultrasound is gaining more attention as a potential measure of success of IOL.

Objective. To assess the role of transvaginal ultrasound measured cervical length (TVS-CL) in predicting the success of IOL.

Methods. A prospective observational study was conducted in the Pretoria Academic Complex. Patients admitted for IOL between 26and 41-weeks' gestation were included in the study regardless of indication. Eligible patients had a modified Bishop score and TVS-CL assessed prior to commencing IOL. IOL was conducted with either mechanical methods, medical methods or a combination thereof. Results. We recruited 150 patients to the study. The modified Bishop score and TVS-CL were highly correlated $(r=-0.74 ; p<0.0001)$. The receiver operating characteristics (ROC) curve analysis and the area under the curve (AUC=0.671) highlighted the poor accuracy of TVS-CL in predicting the success of IOLr compared with the Bishop score. The mean of the TVS-CL was $29.20 \mathrm{~mm}$, with sensitivity of $51 \%$ and specificity of $83 \%$.

Conclusion. TVS-CL is a poor predictor of success of IOL compared with the modified Bishop score. The Bishop score remains valid in a resource-limited setting.

Keywords. induction of labour; transvaginal ultrasound; cervical length; Bishop score.

S Afr J Obstet Gynaecol 2020;27(3):110-114. https://doi/org/10.7196/SAJOG.2020.v27i3.2018

Induction of labour (IOL) is one of the most common interventions in obstetrics. ${ }^{[1]}$ Globally, this procedure is performed in up to $20 \%$ of pregnancies. ${ }^{[2]}$ IOL refers to the use of mechanical or pharmacologic measures to initiate labour. ${ }^{[1]} \mathrm{IOL}$ is indicated when benefits to the mother or the fetus outweigh those of continuing the pregnancy, such as post-dated pregnancy, pre-eclampsia or fetal growth restriction. ${ }^{[1]}$ IOL can be referred to as a 'trial and error' process. Most studies use the criterion of delivery within 24 hours to define the effectiveness of an intervention. ${ }^{[3]}$ However, the success of IOL should rather be defined based on the initiation of labour rather than delivery, which is the endpoint of labour. Failed induction is defined by the National Institute for Health and Care Excellence (NICE) guidelines as failure to establish labour after one cycle of treatment. ${ }^{[4]}$

Several scoring models are used to predict the probability of successful IOL. ${ }^{[1]}$ Widely, the modified Bishop score (henceforth referred to as the Bishop score) is used to predict the success of IOL. The Bishop score was first reported in 1964 and has been used since then as a tool to predict the success of induction. There are limited data regarding the role of the Bishop score to predict the success of IOL. ${ }^{[5]}$ In clinical practice, this method is limited by its subjectivity and interobserver variability. Transvaginal ultrasound measured cervical length (TVS-CL) has therefore been proposed as a better predictor of the success of labour compared with the Bishop score. ${ }^{[6,7]}$ TVS-CL has been linked with the risk of preterm delivery. ${ }^{[8]}$ Cervical shortening as seen on sonar has been proposed as representative of the process of cervical effacement. ${ }^{[8,9]}$ Theoretically, TVS-CL measurement could represent a more accurate assessment of the cervix than digital examination because the supravaginal portion of the cervix usually comprises about 50\% of cervical length, but this is highly variable among individuals. This portion is difficult to assess digitally ${ }^{[8,9]}$ Moreover, ultrasonographic evaluation of cervical characteristics causes less discomfort to patients. ${ }^{[8,9]}$ In experienced hands, TVS is more reproducible and less subjective than digital exam or Bishop score.

The aim of the present study was to examine the value of preinduction TVS-CL in the prediction of success of IOL compared with the clinically determined Bishop score.

\section{Methods}

A multicentre prospective observational study was conducted at two tertiary referral hospitals; namely Kalafong Tertiary Provincial Hospital and Steve Biko Academic Hospital, in Pretoria, South Africa, to assess the accuracy of the TVS-CL in predicting the success of IOL. An average of four patients are admitted daily for IOL at these hospitals.

Patients between 26 and 41 weeks of gestation, with singleton pregnancies in cephalic presentation, and hospitalised for IOL, regardless of the indication, were recruited. Patients with contraindication to a vaginal delivery, multiple pregnancies, previous caesarean sections, or intrauterine fetal demise or fetal congenital anomalies were excluded. Induction methods included 
mechanical induction with a cervical Foley catheter, medical induction with misoprostol, or a combination of mechanical and medical methods. The method of IOL was decided upon based on the Bishop score and maternal characteristics such as grand multiparity and indication of IOL (e.g. preterm pre-labour rupture of membranes).

Patients who consented to participate in the study completed a demographic questionnaire at enrolment into the study. TVS-CL was measured prior to the start of IOL. TVS-CL was measured according to the International Society of Ultrasound in Obstetrics and Gynaecology (ISUOG) guidelines. ${ }^{[10-12]}$ The patient's bladder was emptied, the ultrasound probe was gently inserted into the patient's vagina and guided into the anterior fornix to obtain a sagittal, longaxis image of the entire cervix. Excessive pressure was avoided. The image was enlarged so that the cervix occupied two-thirds (50 - 75\%) of the screen, with both the internal and external os seen clearly. The cervical length was measured along the endocervical canal between the internal and external os. This process was repeated to obtain three sets of image measurements and the shortest best measurement was used. Registrars in the department obtained the measurements. They received training on how to perform a Bishop score and measure cervical length. Interobserver variability was reduced by offering training to all registrars. Posters on how to determine the Bishop score and measure the TVS-CL were posted in the antenatal ward and sonar room as a reminder. Images of the TVS-CL were audited by the principal investigator.

The Bishop score was evaluated at commencement of IOL. Patients were re-assessed after 24 hours to determine their progress of labour. The findings of cervical changes on clinical examination were recorded. A Bishop score $\geq 8$ was regarded as favourable and a score $<8$ was unfavourable, ${ }^{[10]}$ while TVS-CL $<25 \mathrm{~mm}$ was noted to be favourable and $\geq 25 \mathrm{~mm}$ was regarded as unfavourable. ${ }^{[11]} \mathrm{A}$ favourable cervix indicates that a patient has a higher probability of having a successful normal vaginal delivery, while an unfavourable cervix means the contrary. The method of IOL was determined using the Bishop score and maternal characteristics such as grand multiparity and indication of IOL. The successful IOL was defined as a patient being in active labour within 24 hours of initiation of induction agents. Failed induction was defined as failure to establish labour after one completed cycle of treatment.

A convenience sample of 150 participants were included in the study. The correlation between the Bishop score and TVS-CL was assessed using the Bishop score as the gold standard. The sensitivity, specificity, and positive and negative predictive values (PPV and NPV) were determined as well as the positive and negative likelihood ratios (LR+ and LR-) of the TVS-CL at predetermined cut-off values $(<$ or $>25 \mathrm{~mm})$ for the prediction of success of IOL. The accuracy of the performance of the TVS-CL was evaluated with receiver operating characteristic (ROC) curves.

Ethical approval for this study was obtained from the University of Pretoria, Faculty of Health Sciences Research Ethics Committee (ref. no. 582/2018). Informed consent was obtained from the patients prior to enrolment in the study.

\section{Results}

A total of 150 patients were recruited into the present study. The characteristics of the patients are presented in Table 1.

The average (standard deviation (SD)) maternal age was 30.55 (6.45) years, the average (SD) mid-upper arm circumference was
$30.97(4.16) \mathrm{cm}$, and the average (SD) estimated gestational age was 37.97 (1.84) weeks. Hypertensive disorders of pregnancy were the most common indication of IOL $(38 \% ; n=57)$.

The average (SD) Bishop score was 6.61 (1.81), which is unfavourable. Similarly, the average (SD) TVS-CL was 29.2 (7.60) $\mathrm{mm}$, which was also unfavourable.

Table 1. Characteristics of the population $(N=150)$

\begin{tabular}{ll}
\hline Characteristics of patients & Mean $(\text { Range; SD })^{\star}$ \\
\hline Age (years) & $30.55(16-44 ; 6.45)$ \\
Gravidity & $2.64(1-8 ; 1.45)$ \\
Parity & $1.25(0-5 ; 1.19)$ \\
Gestational age (weeks) & $37.97(32-42 ; 1.84)$ \\
Weight $(\mathrm{kg})$ & $81.31(46-160 ; 19.82)$ \\
Mid-upper arm circumference $(\mathrm{cm})$ & $30.97(21-46 ; 4.16)$ \\
Haemoglobin (g/dL) & $11.57(9-15 ; 1.14)$ \\
Indications for IOL, $n$ (\%) & \\
Post dates & $22(14.7)$ \\
HPT & $57(38.0)$ \\
PPROM or PROM & $17(11.3)$ \\
GDM & $31(20.7)$ \\
Prev IUD & $5(3.3)$ \\
IUGR & $5(3.3)$ \\
Others & $13(8.7)$ \\
GDM = gestational diabetes; HPT = hypertension; IOL = induction of labour; \\
IUD = intrauterine fetal demise; IUGR; =intrauterine fetal growth restriction; \\
PPROM = preterm prelabour rupture of membranes; PROM = preterm rupture of membranes. \\
*Unless otherwise specified.
\end{tabular}

Table 2. Characteristics of the cervical findings $(N=150)$

\begin{tabular}{lll}
\hline & $\begin{array}{l}\text { Modified Bishop score, } \\
\text { mean (SD)* }\end{array}$ & $\begin{array}{l}\text { TVS-CL, } \\
\text { mean (SD)* }\end{array}$ \\
\hline Cervical length $(\mathrm{mm})$ & $17.4(0.60)$ & $29.2(7.60)$ \\
Bishop score & $6.61(1.81)$ & - \\
Favourable, ${ }^{\dagger} n(\%)$ & $61(40.7)$ & $57(38)$ \\
Unfavourable ${ }^{\dagger} n(\%)$ & $89(59.3)$ & $93(62)$ \\
& \\
&
\end{tabular}

Table 3. Characteristics of IOL and obstetrics outcomes $(N=150)$

\begin{tabular}{ll}
\hline Characteristics of patients & $\boldsymbol{n}(\%)^{\star}$ \\
\hline Mode of induction & $67(44.7)$ \\
$\quad$ Medical & $2(1.3)$ \\
Mechanical & $81(54.0)$ \\
$\quad$ Both & $111(74.0)$ \\
Time to delivery (hours) & $39(26.0)$ \\
$\quad<24$ & \\
$>24$ & $97(64.7)$ \\
Mode of delivery & $53(35.3)$ \\
$\quad$ NVD & $27(50.9)$ \\
C/D & $18(34.0)$ \\
Indication of C/D & $6(11.3)$ \\
Fetal distress & $2(3.8)$ \\
Failed IOL & $3060.57 ;$ \\
Cephalopelvic disproportion & $(1719-4$ 310; 555.69) \\
Other & \\
Birthweight (g), mean (range; SD) & \\
&
\end{tabular}


Half of the women (54\%; $n=81)$ were induced with a combination of misoprostol and mechanical induction with a cervical Foley's catheter. Despite most women having an unfavourable Bishop score and TVS-CL, $64 \%$ had a successful IOL. The time from IOL to delivery was $<24$ hours in $74 \%(n=111)$ of the patients. The caesarean delivery rate was $35.3 \%$, with half of these due to fetal distress. Moreover, only 34\% $(n=18)$ of caesarean deliveries were due to failed IOL.

The correlation between the TVS-CL measurement and Bishop score is illustrated in Fig. 1. There was a significant relationship ( $r=-0.74 ; p<0.0001)$ between the TVS-CL and the Bishop score. The area under the ROC curve (AUC) was 0.0671 (Fig. 2). The mean (SD) cervical length was 29.2 (7.60) $\mathrm{mm}$, with a sensitivity of $51 \%$ and specificity of $83 \%$ based on the ROC analysis (Table 4 ).

About a quarter of women $(26 \% ; n=39)$ had a favourable cervix indicated by the TVS-CL threshold of $25 \mathrm{~mm}$ (Table 4). The PPV was $51.28 \%$ and the NPV of $82.88 \%$. The LR+ is 3.00 and LR- is 0.59 , meaning that TVS-CL is a poor predictor for success of IOL. Table 5 illustrates the factors affecting success of IOL.

\section{Discussion}

The main aim of the present study was to evaluate the accuracy of TVS-CL in predicting the success of IOL. Additionally, the performance of TVS-CL was compared with the Bishop score as a predictor of the success of IOL. Although we found that there was a high corelation between the two tests, the findings of the present study do not support the use of TVS-CL as an independent predictor of the success of IOL. We also showed that the Bishop score is a better predictor of success of IOL despite the known interobserver variability for the Bishop score.

A study by Gonen et al. ${ }^{[13]}$ demonstrated that once the Bishop score was known, the addition of the ultrasonographic variables did not improve the ability to predict the outcome of the IOL. Similarly, Groeneveld et al. ${ }^{[14]}$ also concluded that the Bishop score is a predictor of successful vaginal delivery and that ultrasound measurement of CL does not contribute to the prediction of successful vaginal delivery. On the contrary, a study by Rane et al.$^{[15]}$ found that using the TVS-CL to predict the success of IOL had a sensitivity of $89 \%$, which was superior to the Bishop score that had a sensitivity of $65 \%{ }^{[17]}$ In addition, Pandis et al. ${ }^{[16]}$ demonstrated that CL performed better than the Bishop score in the prediction of vaginal delivery. These differing findings might be attributed to the heterogenous population studied and the study design.

In the present study, it is evident that the supravaginal portion of the cervix is difficult to assess digitally as the cervical length was always longer on transvaginal ultrasound as compared with effacement measured digitally. While this has been an argument for the use of TVS-CL, it has not been consistently proven to be clinically significant. ${ }^{[8,9,17]}$

Maternal characteristics in the present study did not affect the outcome of IOL. Observational studies indicate that obese women are 1.6 times more likely to fail IOL than women of normal body mass index. ${ }^{[18]}$ The findings in the present study indicate otherwise probably owing to a small sample size. This may suggest that there is a need for further investigations on this topic, especially in our population, where the fat distribution (central v. pelvic-femoral) may possibly be a factor.

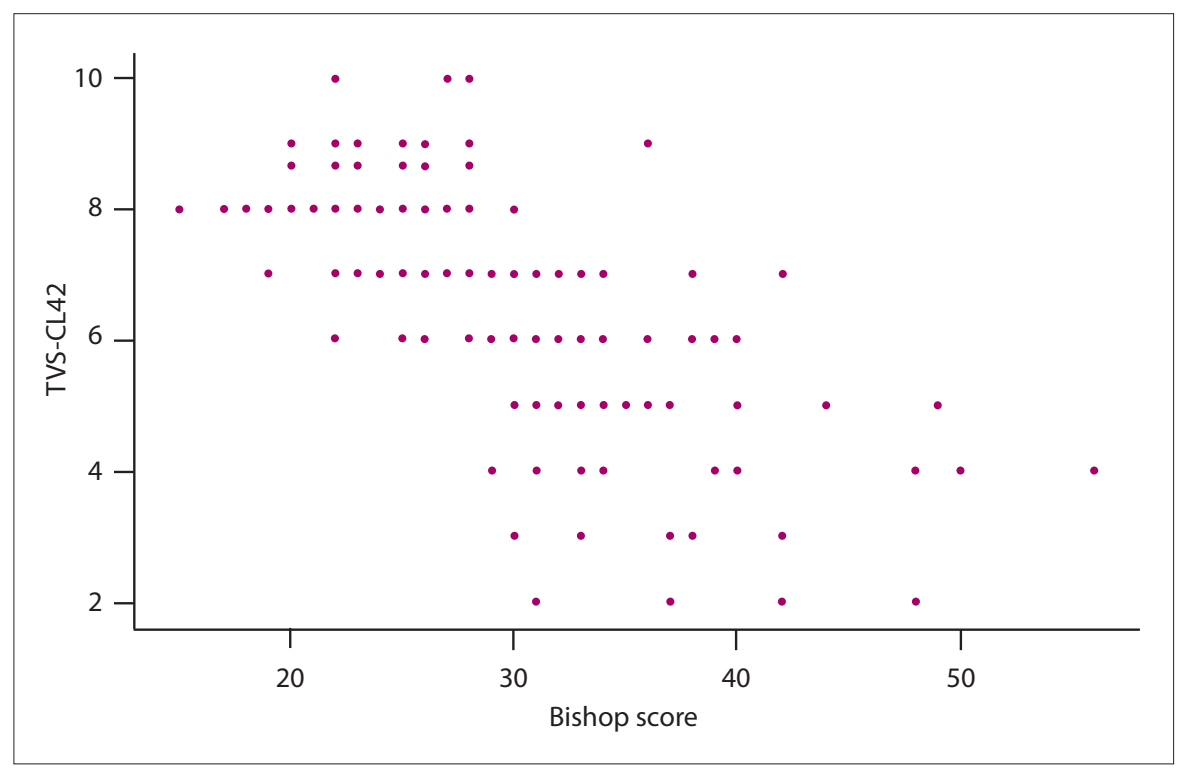

Fig. 1. Correlation between the Bishop score and TVS-CL in predicting the success of IOL $(\mathrm{r}=-0.74)$. (TVS-CL = transvaginal ultrasound measured cervical length; $I O L=$ induction of labour).

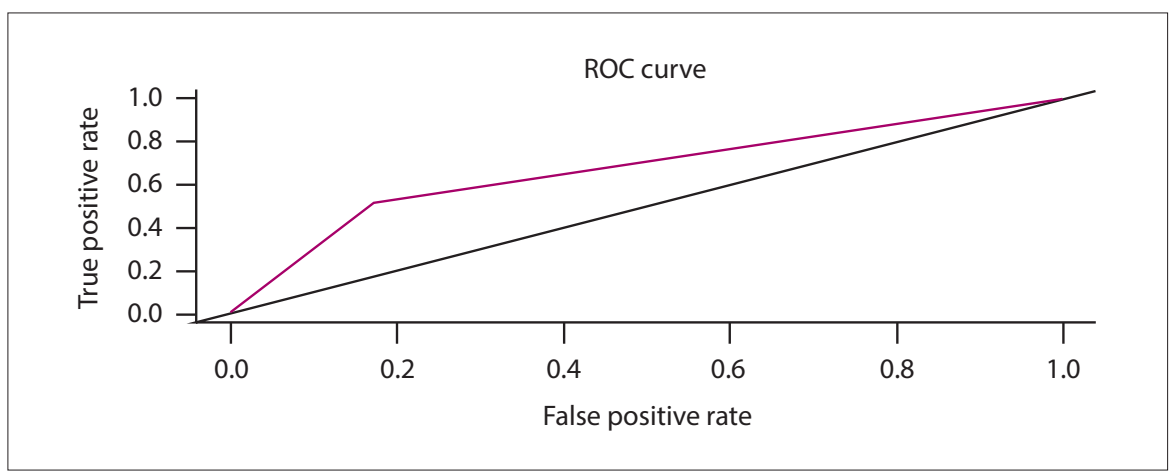

Fig. 2. ROC curve demonstrating the ability of TVS-CL to predict the success of IOL (AUC 0.671). (ROC = receiver operating characteristic; $A U C=$ area under the curve).

Table 4. Sensitivity, specificity, LR+, LR-, PPV, and NPV for Bishop score and TVS-CL

\begin{tabular}{|c|c|c|c|c|c|c|}
\hline & Sensitivity (\%) & Specificity (\%) & LR+ & LR- & PPV & NPV \\
\hline Modified Bishop score & 99 & 77 & 3.94 & 0.10 & 58.06 & 96.59 \\
\hline TVS-CL & 51 & 83 & 3.00 & 0.59 & 51.28 & 82.88 \\
\hline
\end{tabular}


RESEARCH

Table 5. Factors affecting the success of IOL

\begin{tabular}{|c|c|c|c|c|c|}
\hline Characteristics & Successful IOL, $\boldsymbol{n}(\%)$ & Failed IOL, $n(\%)$ & OR & $95 \% \mathrm{CI}$ & $p$-value \\
\hline \multicolumn{6}{|l|}{ GA (weeks) } \\
\hline$\leq 37$ & $22(25.9)$ & $10(32.3)$ & 0.77 & $0.30-1.80$ & \multirow[t]{2}{*}{0.4984} \\
\hline$>37$ & $63(74.1)$ & $21(67.7)$ & 1.36 & $0.56-3.34$ & \\
\hline \multicolumn{6}{|l|}{ Obesity } \\
\hline MUAC $\leq 33 \mathrm{~cm}$ & $60(70.6)$ & $22(71.0)$ & 0.98 & $0.40-2.43$ & \multirow[t]{2}{*}{0.9684} \\
\hline MUAC $>33 \mathrm{~cm}$ & $25(29.4)$ & $9(29.0)$ & 1.02 & $0.41-2.51$ & \\
\hline \multicolumn{6}{|l|}{ Parity } \\
\hline Nullipara & $22(25.9)$ & $11(35.5)$ & 0.63 & $0.26-1.53$ & \multirow[t]{2}{*}{0.3125} \\
\hline$>1$ & $63(74.1)$ & $20(64.5)$ & 1.56 & $0.65-3.80$ & \\
\hline \multicolumn{6}{|l|}{ Indications of IOL } \\
\hline Postdate & $14(16.5)$ & $1(3.2)$ & - & - & - \\
\hline HDP & $27(31.8)$ & $18(58.1)$ & - & - & - \\
\hline PPROM/PROM & $10(11.8)$ & $3(9.7)$ & - & - & - \\
\hline GDM & $18(21.2)$ & $7(22.6)$ & - & - & - \\
\hline Other & $16(18.8)$ & $2(6.5)$ & - & - & - \\
\hline
\end{tabular}

One of the interesting findings from our study was that the Bishop score was better than TVS-CL but still a poor predictive tool for the success of labour. This finding highlights the question of whether there is a role for these tools in modern obstetrics. Despite knowing that a woman has an unfavourable cervix, it is unlikely to change the management plan, more especially with a global consensus on reducing primary caesarean delivery. ${ }^{[19]}$

Several groups have evaluated the usefulness of transvaginal ultrasound in the prediction of successful IOL and all have reached the same conclusion that $\mathrm{CL}$ is a good predictor of the duration of labour. Yet, it remains unknown whether it is clinically useful, or whether it could replace the Bishop score, or whether both methods should be used together. ${ }^{[14,20]}$ The American College of Obstetrics and Gynecology (ACOG) recommends that the status of the cervix be determined by the Bishop pelvic scoring system. ${ }^{[17]}$ Currently, transvaginal CL is not endorsed by ACOG as a method of assessing the likelihood of success or failure of IOL owing to limited data.

Based on the findings from the present study, the Bishop score appears to be superior to the CL measurement. The Bishop score is a simple, cheap method; therefore, it is a very useful method in low-resource settings where ultrasound machines are not widely accessible. Considering the global consensus to decrease caesarean sections in non-emergency cases, a trial of IOL must always be considered.

\section{Study strengths and limitations}

The strengths of the present study are the pragmatic study context, multicentre data collection and use of readily available tools. The limitations of the study include the small sample size and interobserver variability. We did put measures in place to reduce interobserver variability in the present study. There is controversy with the current findings on this subject as guidelines are contradictory on whether or not transvaginal CL is useful in predicting the success of IOL; thus, randomised control studies with larger numbers of women are recommended for future research.

\section{Conclusion}

The present study showed that TVS-CL is a poor predictor for the success of IOL compared with the modified Bishop score.
Declaration. This study was done in partial fulfilment of requirements for a MMed (O\&G) degree.

Acknowledgements. We are grateful to the women who participated in the study, and the hospital staff and nurses of the Steve Biko and Kalafong hospitals who supported the study.

Author contributions. AA developed the research questions and protocol, collected and analysed data collection, and wrote the manuscript. TC performed statistical analysis. SA conceived and designed the study and revised the manuscript. All authors approved the final version of the manuscript for publication.

Funding. None.

Conflicts of interest. None.

1. Verhoeven CJM, Opmeer BC, Oei SG, Latour V, van der Post JAM, Mol BWJ. Transvaginal sonographic assessment of cervical length and wedging for predicting outcome of labor induction at term: A systematic review and meta-analysis. Ultrasound Obstet Gynaecol 2013;42(5):500-508 https://doi.org/10.1002/uog.12467

2. Laencina AM, Gómez S, Francisco G, et al. Comparison of ultrasonographic cervical length and the Bishop score in predicting successful labor induction. Acta Obstet Gynaecol Scand 2007;86(7): 799-804. https://doi.org/10.1080/00016340701409858

3. Chodankar R, Sood A, Gupta J. An overview of the past, current and future trends for cervical ripening in induction of labour. Obstetr Gynaecol 2017;19:219-226. https://doi.org/10.1111/tog.12395

4. Ryan RM, McCarthy FP. Induction of labour. Obstetr Gynaecol Reprod Med 2016;26(10):304-305. https://doi.org/10.1016/j.ogrm.2016.07.005

5. Reece AE, Hobbins JC. Clinical Obstetrics: The fetus and mother. 3rd ed. Hoboken: Wiley-Blackwell, 2007.

6. Pereira S, Frick AP, Poon LC, Zamprakou A, Nicolaides KH. Successful induction of labor: Prediction by preinduction cervical length, angle of progression and cervical elastography. Ultrasound Obstet by preinduction cervical length, angle of progression and cervics
Gynaecol 2014;44(4):468-475. https://doi.org/10.1002/uog.13411

7. Tanir HM, Sener T, Yildiz Z. Digital and transvaginal ultrasound cervical assessment for prediction of successful labour induction. Int J Gynaecol Obstetr 2008;100(1):52-55. https://doi.org/10.1016/j. ijgo.2007.07.014

8. Keepanasseril A, Suri V, Bagga R, Aggarwal N. Pre-induction sonographic assessment of the cervix in the prediction of successful induction of labour in nulliparous women. Aust N Z J Obstetr Gynaecol 2007;47(5):389-393. https://doi.org/10.1111/j.1479-828x.2007.00762.x

9. Bartha JL, Romero-Carmona R, Martínez-del-Fresno P, Comino-Delgado R. Bishop score and transvaginal ultrasound for preinduction cervical assessment: A randomised clinical trial. Ultrasound Obstet Gynaecol 2005;25(2):155-159. https://doi.org/10.1002/uog.1813

10. Laughon SK, Zhang J, Troendle J, Sun L, Reddy UM. Using a simplied Bishop score to predict vaginal delivery. Obstet Gynaecol 2011;117(4):805-811. https://doi.org/10.1097/aog.0b013e3182114ad2

11. Ibrahim AA, Mohannad LA. Sonographic assessment of the cervical length before induction of labour. Asian Pacific J Reprod 2012;1(4):253-257.

12. Kagan KO, Sonek J. How to measure cervical length. Ultrasound Obstet Gynaecol 2015;45:358-362. https://doi.org/10.1002/uog.14742

13. Gonen R, Degani S, Ron A. Prediction of successful induction of labour: Comparison of transvaginal ultrasonography and the Bishop score. Eur J Ultrasound 1998;7(3):183-187. https://doi.org/10.1016/ s0929-8266(98)00042-1

14. Groeneveld YJB, Bohnen AM, van Heusden AM. Cervical length measured by transvaginal ultrasonography v. Bishop score to predict successful labour induction in term pregnancies. Facts Views Vis Obgyn 2010;2(3):187-193

15. Rane SM, Guirgis RR, Higgins B, Nicolaides KH. The value of ultrasound in the prediction of successful induction of labour. Ultrasound Obstet Gynaecol 2004;24:538-549. https://doi. org/10.1002/uog.1100 


\section{RESEARCH}

16. Pandis GK, Papageorghiou AT, Ramanathan VG. Pre-induction sonographic measurement of cervical length in the prediction of successful induction of labour. Ultrasound Obstet Gynaecol 2001;18(6):623-628. https://doi.org/10.1046/j.0960-7692.2001.00580.x

17. American College of Obstetricians and Gynaecologists (ACOG). Induction of labour. Clinical management guidelines for obstetrician - Gynaecologist number 107. ACOG practice bulletin, 2009. Obstet Gynecol 2009;114(2):386-397. https://doi:10.1097/AOG.0b013e3181b48ef5

18. Nugent R. RCOG 2018: Obese pregnant women are more likely to experience difficult induction of labour. https://www.practiceupdate.com/content/rcognbsp2018-obese-pregnant-women-aremore-likely-to-experience-difficult-induction-of-labor/66511 (accessed 11 November 2020).
19. American College of Obstetricians and Gynecologists (ACOG). Safe prevention of the primary caesarean delivery. Obstetric Care Consensus number 1. J Perinat Educ 2014;23(3):115-118. https://doi:10.1891/1058-1243.23.3.115

20. Ware V, Raynor BD. Transvaginal ultrasonographic cervical measurement as a predictor of successful labour induction. Am J Obstet Gynecol 2000;182(5):1030-1032. https://doi.org/10.1067/ mob.2000.105399

Accepted 6 July 2021 